\title{
Ipsilesional High Frequency Repetitive Transcranial Magnetic Stimulation Add-On Therapy Improved Diffusion Parameters of Stroke Patients with Motor Dysfunction: A Preliminary DTI Study
}

\author{
Zhiwei Guo, ${ }^{1}$ Yu Jin, ${ }^{1}$ Haitao Peng, ${ }^{1}$ Guoqiang Xing, ${ }^{1,2}$ Xiang Liao, ${ }^{1}$ Yunfeng Wang, ${ }^{3}$ \\ Huaping Chen, ${ }^{1}$ Bin He, ${ }^{1}$ Morgan A. McClure, ${ }^{1}$ and Qiwen $\mathrm{Mu}^{1,4}$ \\ ${ }^{1}$ Department of Imaging and Imaging Institute of Rehabilitation and Development of Brain Function, \\ The Second Clinical Medical College of North Sichuan Medical College, Nanchong Central Hospital, Nanchong 637000, China \\ ${ }^{2}$ Lotus Biotech.Com LLC., John Hopkins University-MCC, Rockville, MD, USA \\ ${ }^{3}$ Department of Neurology, The Second Clinical Medical College of North Sichuan Medical College, \\ Nanchong Central Hospital, Nanchong 637000, China \\ ${ }^{4}$ Peking University Third Hospital, Beijing, China
}

Correspondence should be addressed to Qiwen Mu; muqiwen99@yahoo.com

Received 7 July 2016; Accepted 31 August 2016

Academic Editor: Lin Ai

Copyright (C) 2016 Zhiwei Guo et al. This is an open access article distributed under the Creative Commons Attribution License, which permits unrestricted use, distribution, and reproduction in any medium, provided the original work is properly cited.

\begin{abstract}
Purpose. The aim of this study was to evaluate the effects of high frequency repetitive transcranial magnetic stimulation (HF-rTMS) on stroke patients with motor dysfunction and to investigate the underlying neural mechanism. Methods. Fifteen stroke patients were assigned to the rTMS treatment (RT) group and conventional treatment (CT) group. Patients in the RT received $10 \mathrm{~Hz}$ rTMS stimulation on the ipsilesional primary motor cortex for 10 days plus conventional treatment of CT, which consisted of acupuncture and antiplatelet aggregation medication. Difference in fractional anisotropy (FA) between pretreatment and posttreatment and between two groups was determined. Correlations between FA values and neurological assessments were also calculated. Results. Both groups significantly improved the neurological function after treatment. rTMS-treated patients showed better improvement in Fugl-Meyer Assessment (FMA) score and increased FA value in motor-related white matter and gray matter cortices compared with CT-treated patients and pretreatment status. Besides, the increased FA value in the ipsilesional posterior limb of the internal capsule in RT group was significantly correlated with the improved FMA score. Significance. HF-rTMS could be a supplement therapy to CT in improving motor recovery in patients with stroke. And this benefit effect may be achieved through modulating the ipsilesional corticospinal tracts and motor-related gray matter cortices.
\end{abstract}

\section{Introduction}

Stroke is the major cause of adult disability worldwide. Up to $80 \%$ of the stroke patients endure motor deficits that severely lowers the quality of their daily lives $[1,2]$. Most of the stroke survivors could obtain a certain degree of motor improvement after various therapies, including medication, acupuncture, movement training, and other types of interventions. So far, however, the effectiveness of these interventions remains unsatisfactory.
The neural mechanisms of motor recovery following stroke remain unknown. Recent studies indicate that the functional plasticity and structural remodeling of white microstructure could underlie the poststroke recovery process [3-6]. Transcranial magnetic stimulation (TMS) is a safe, painless, and noninvasive strategy that was first reported by Barker et al. in 1985 [7, 8]. Repetitive TMS (rTMS) is a new method that can alert activity in cortex and induce lasting effects on neuroplasticity in the cortex, and it has been used to treat depression, Parkinson's disease, stroke, and other 
neurological diseases in recent years. The rTMS therapy for motor recovery following stroke aims to augment neural plasticity and improve motor function based on the interhemispheric competition model, which states that inhibitory rTMS on contralesional hemisphere increases excitability in the ipsilesional motor cortex by reducing excessive interhemispheric inhibition from the contralesional motor cortex [9-11], whereas excitatory rTMS over the affected hemisphere directly increases the excitability of the ipsilesional motor cortex [11-14].

Previous plastic studies of rTMS had focused mainly on functional brain mapping. Currently, diffusion tensor imaging (DTI) has been widely used as a noninvasive tool to investigate and measure the integrity of white matter in vivo [15]. Fractional anisotropy (FA), one of DTI parameters, can quantify the degree of water diffusion and reliably visualize the microstructural status, but it is susceptible to axonal myelination as well as density and orientational coherence [16]. Reduced FA has been reported in Wallerian degeneration or destruction of white matter integrity [17] and in people with motor impairments [18]. Several DTI-based studies that evaluated the FA values along the ipsilesional corticospinal tracts (CST) suggest a decreased FA value following stroke [19-21]. After the interventions of different treatments, the progressively increased FA values in the ipsilesional CST were found positively correlated with the functional recovery of the stroke patients as measured by the Fugl-Meyer Assessment (FMA) $[4,5,22]$. Several related studies also reported that the stroke motor recovery is positively and significantly associated with the changes of FA in the contralesional hemisphere after unilateral infract [23-25]. Besides, previous low frequency rTMS studies also have shown obvious effects in improving motor function via inhibiting contralesional motor cortex and exciting the ipsilesional cortex [26, 27]. To date the research on rTMS-induced white matter modification after stroke is limited, and it is not clear if high frequency rTMS could also lead to an improvement in motor function and remodeling of white microstructures in stroke patients with motor dysfunction.

The present study aimed at determining whether high frequency rTMS-induced excitability of the ipsilesional hemisphere would cause plastic changes in the microstructure of white matter of stroke patients with motor dysfunction and the correlation with motor recovery.

\section{Methods}

2.1. Participants. Fifteen acute ischemic stroke patients with left hemisphere infarctions were recruited for this study from March to December 2015 at the Second Clinical Medical College of North Sichuan Medical College, Nanchong, China, with the following inclusion criteria: (1) first-ever stroke patients with a unilateral hemisphere infarct within seven days of onset; (2) the lesion being subcortical with its focal point confirmed by diffusion weighted imaging (DWI); (3) National Institutes of Health Stroke Scale (NIHSS) scores $\geq 2$; (4) mild to moderate motor impairment lasting at least 48 hours; (5) age from 50 to 80 years; and (6) being without seizure, dysgnosia, psychosis, or other coexistent neurological/psychiatric disease. All study procedures were conducted in accordance with the Helsinki Declaration of 1975 and were approved by Institutional Review Board of the North Sichuan Medical College. Informed consent was obtained from all participants prior to enrollment into the study.

A total of 15 patients were assigned to two groups: 7 to the rTMS treatment (RT) group and 8 to the conventional treatment (CT) group. The patients in the RT group received $10 \mathrm{~Hz}$ rTMS treatment over the ipsilesional motor cortex for 10 days plus conventional treatment, whereas patients in CT group underwent conventional treatment including acupuncture and antiplatelet aggregation drugs medication.

2.2. MRI Procedure and Neurological Evaluation. Each patient received the neurological functional assessments and MRI scans two times: prior to the first rTMS session and immediately after the end of rTMS treatments (10 days of rTMS stimulation). All patients received the same medical therapy including anticoagulant (low molecular weight heparin or aspirin), brain protection (piracetam), and blood circulation protection (Salvia miltiorrhiza).

2.3. Clinical Assessment. All patients were assessed by FuglMeyer Assessment (FMA), NIHSS, and Barthel Index scale (BI) to evaluate the severity of stroke and functional disability by trained and experienced neurologists before MRI examination. The FMA measurement consists of the motor function assessment items on upper limb and lower limb. BI is often used to evaluate the activity ability of daily living of stroke patient. For both of FMA and BI scales, a higher score reveals a better motor function or activity ability. On the contrary, a higher score of NIHSS, a comprehensive assessment to estimate the degree of neurological impairment, reflects a more serious stroke-related disability. Behavioral assessment and MRI examination were both conducted on the same day. To minimize operator-dependent bias, the neurologists were blinded to the patient grouping. The differences in clinical assessment scores between the RT and CT groups and before the first session and after the end of treatment were analysed by using the SPSS 22.0 software (Statistical Package for Social Sciences, Chicago, IL, USA). The results were considered significant at $p<0.05$.

2.4. Resting Motor Threshold. For the rTMS treatment, the TMS coil was placed on the ipsilesional motor cortex. To determine the stimulation intensity, the resting motor threshold (RMT), defined as the minimal output of stimulation that could evoke muscle twitch of the contralateral first dorsal interosseous (FDI) or elicited a motor evoked potential (MEP) of at least an amplitude of $50 \mu \mathrm{V}$ in at least half of 10 consecutive stimuli recorded by electromyography [28], was determined for each patient by connecting the rTMS stimulator to an electromyogram apparatus (Dantec Keypoint System, Skovlunde, Denmark). The MEP signal was recorded from the surface $\mathrm{Ag} / \mathrm{AgCI}$ electrodes placed on the FDI hand muscles. 
2.5. rTMS Protocols. Patients in the RT group received 10 daily sessions of rTMS over the hand area of the ipsilesional primary motor cortex (M1) for a duration of 15 minutes using a Mag Pro butterfly-shape coil stimulator (MagVenture, Lucernemarken, Denmark). Each session of rTMS involved 30 trains of 50 pulses with 25 -second intervals at $10 \mathrm{~Hz}$ and 90\% RMT (total 1500 pulses/day). The stimulated motor cortex was determined and defined as the location that could elicit muscle twitch and the largest MEP of the contralateral FDI. As the ipsilesional hemisphere remained nonresponsive to TMS stimulation, the exact site of stimulation was defined as the location homologous to the contralesional motor cortex. Besides, during the rTMS treatment, the coil was positioned tangentially to the scalp of the stimulation target at a $45^{\circ}$ angle from the midsagittal plane.

2.6. Acupuncture Strategy. Acupuncture was performed at the bilateral Fengchi acupoint and ipsilesional Baihui, Xuanzhong, Quchi, Hegu, Zusanli, and Sanyinjiao acupoints, with Baihui and Fengchi forward flat spines 0.5-1.5 inch, obliquely to the tip of the nose direction of the wind pool, and the remaining acupoints down to levels of $0.8-2.0$ inches. The twisting angle was less than 90 degrees. The acupuncture treatment was performed by experienced and licensed acupuncturists. Each patient received thirty minutes of acupuncture treatment per day following rTMS treatment. Patients in both the RT and CT groups were also given antiplatelet aggregation drugs to improve blood circulation.

2.7. MRI Acquisition. All MRI data were acquired on a GE Signa HDxt 1.5 Tesla MR scanner (General Electric Medical System, Milwaukee, WI, USA) by using an 8-channel head coil. The DTI acquisition was performed by using a singleshot echo-planar imaging (EPI) sequence with the following parameters: TR/TE $=8500 / 96 \mathrm{~ms}$, flip angle $=90^{\circ}$, field of view $=240 \mathrm{~mm} \times 240 \mathrm{~mm}$, matrix $=256 \times 256$, voxel sizes $=$ $0.94 \times 0.94 \times 5.0 \mathrm{~mm}^{3}, 32$ axial slices with no gap, and acquisition time $=4$ minutes and 50 seconds. The diffusion sensitive gradients were applied along 30 noncollinear directions with $b$ value $=1000 \mathrm{~s} / \mathrm{mm}^{2}$ to obtain the weighted images and one unweighted B0 image with $b$ value $=0 \mathrm{~s} / \mathrm{mm}^{2}$.

Along with the diffusion tensor imaging scan, high resolution anatomical T1-weighted images were also acquired for each subject using a three-dimensional-spoiled gradient recalled $(3 \mathrm{D}-\mathrm{SPGR})$ sequence: $\mathrm{TR} / \mathrm{TE}=9.1 / 2.9 \mathrm{~ms}$, flip angle $=20^{\circ}$, field of view $=240 \mathrm{~mm} \times 240 \mathrm{~mm}$, matrix $=256 \times 256$, voxel sizes $=0.94 \times 0.94 \times 1.2 \mathrm{~mm}^{3}$, and 124 slices with no gap.

2.8. Image Processing. SPM8 software (statistical parametric mapping, http://www.fil.ion.ucl.ac.uk/spm/) was used for preprocessing of DTI datasets to remove the head motion by aligning 30 diffusion weighted images to B0 image. Eddy current distortions were corrected by affine registration to the reference B0 image. Whole brain fiber tracking and reconstruction of the diffusion parameter images were evaluated in the DTI native space using interpolated streamline propagation algorithm with Diffusion Toolkit Software (http://trackvis.org/). Three eigenvalues $(\lambda 1, \lambda 2$, and $\lambda 3)$ and the maps of $\mathrm{MD}$ and FA were produced for each subject.

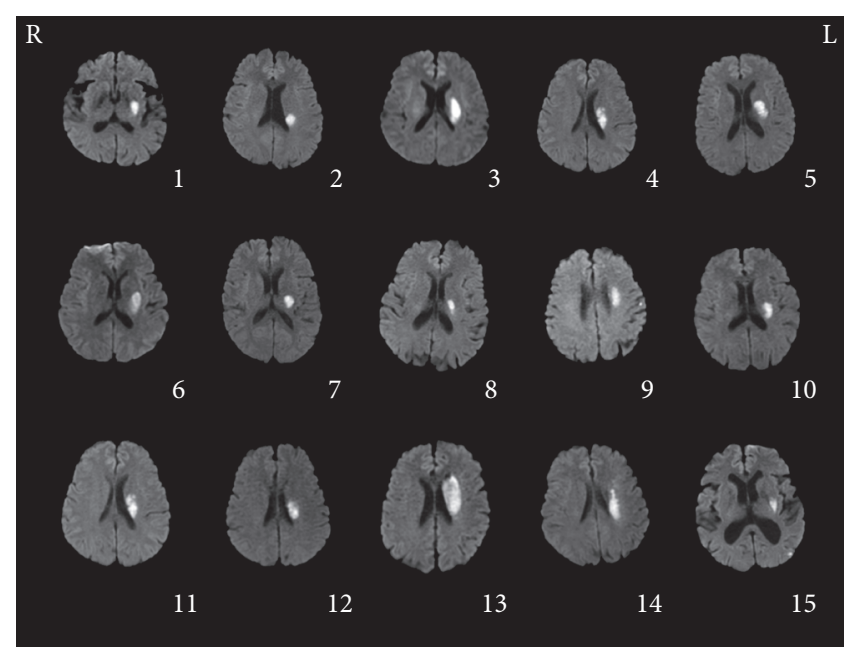

FIGURE 1: Individual diffusion weighted images in the axial view. The panel shows the slice with maximum infarct volume. Each subject is coded by the same serial number as the first row in Table 1 .

During fiber tracking, if the FA value was lower than 0.2 or if the angle was less than $40^{\circ}$, the path tracking was stopped. All of the maps were normalized to the Montreal Neurological Institute $(\mathrm{MNI})$ coordinate system and reinterpolated into isotropic voxels of $3.0 \mathrm{~mm} \times 3.0 \mathrm{~mm} \times 3.0 \mathrm{~mm}$. Finally, to improve the signal-to-noise ratio of the maps, an isotropic Gaussian kernel (FWHM $=8 \mathrm{~mm}$ ) was applied to complete the spatial smooth filtering. To detect the alteration of MD and FA values after the rTMS treatment, a paired $t$-test was adopted with a statistical significant level of $p<0.05$. The two-sample $t$-test was also used to compare the images between the patients from RT and CT groups. To minimize the possible impact on the findings, age, gender, and duration of stroke were used as covariates in all statistical analyses.

2.9. Correlation Analysis. After comparing the FA maps between pre- and post-rTMS treatments, the motor-related brain regions, which revealed significant differences, were selected as regions of interest (ROI) to investigate the potential relationships between the alteration of diffusion parameters in these ROIs and clinical assessment improvement. Each ROI was defined as a cluster composed of 27 voxels around the peak coordinate of the difference area. For each ROI, the extracted values were manually checked and confirmed before the average value of the 27 voxels was obtained. Finally, Pearson correlation analysis was conducted between the diffusion parameters and clinical assessments including FM and BI to estimate their homogeneity.

\section{Results}

Table 1 shows the demographic and clinical characteristics of the included stroke patients. No significant differences in age, gender, type of stroke, duration, or baseline behavioral scores were observed between the RT and CT groups at baseline. Figure 1 illustrates the lesion location from the slice of maximum infarct volume on DWI images for each 


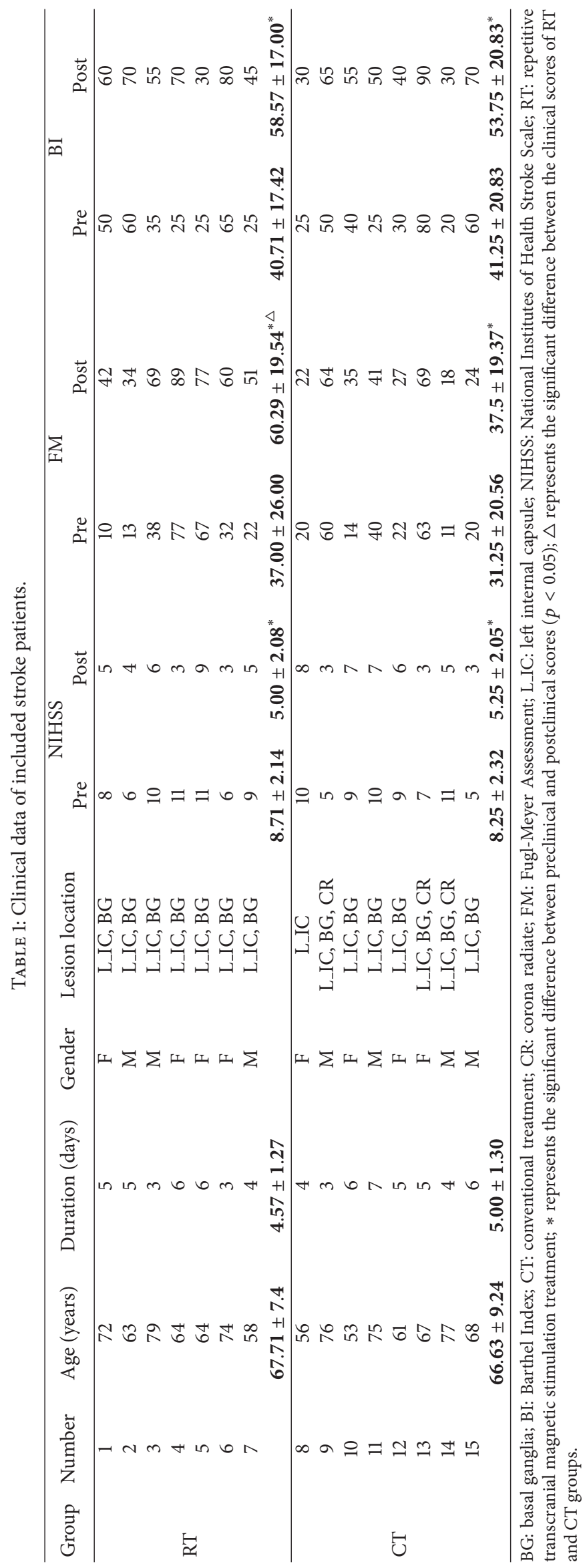


TABLE 2: Brain regions with significant clusters and peak voxel coordinates showing FA difference between pre- and post-rTMS treatment.

\begin{tabular}{lccc}
\hline Brain region & $\begin{array}{c}\text { MNI coordinates } \\
(x, y, z)\end{array}$ & $t$ value & $\begin{array}{c}\text { Voxel } \\
\text { number }\end{array}$ \\
\hline Post $>$ pre & & & \\
$\quad$ Supp_motor_area_R & $7,-23,60$ & 4.89 & 77 \\
Frontal_mid_R & $36,10,51$ & 3.18 & 48 \\
Precentral_L & $-38,-2,48$ & 4.67 & 55 \\
Precentral_R & $27,-20,54$ & 4.22 & 73 \\
Thalamus_L & $-23,-23,6$ & 7.56 & 39 \\
Temporal_inf_L & $-59,-20,-30$ & 6.89 & 31 \\
\hline Post $<$ pre & & & \\
Precuneus_R & $5,-68,53$ & -4.74 & 32 \\
Parietal_sup_R & $27,-49,51$ & -5.58 & 54 \\
Angular_L & $-44,-61,48$ & -2.84 & 28 \\
\hline
\end{tabular}

MNI: Montreal Neurological Institute; L: left; R: right.

participant. All of the patients completed the 10 treatment sessions without reporting any adverse effects.

3.1. Behavioral Outcomes. Table 1 shows the descriptive pretreatment and posttreatment data for all clinical measures for both RT and CT groups. NIHSS, FMA, and BI scores showed significant changes after the treatment in both groups (NIHSS: RT: $p=0.003$; CT: $p=0.001$; FMA: RT: $p=$ 0.001; CT: $p=0.026$; BI: RT: $p=0.012$, CT: $p=0.001$ ). Additionally, the increase in FMA was significantly greater in the RT than in the CT group after the treatment $(p=0.041)$.

3.2. Factional Anisotropy Improvement. Paired comparisons between pre- and post-rTMS conditions revealed that the quantitative diffusion FA values of patients in the RT had increased significantly in the ipsilesional posterior limb of internal capsule (PLIC), M1, contralesional supplementary motor area (SMA), middle frontal gyrus (MFG), bilateral CST at the level of corona radiate (CR), and contralesional PLIC after 10 days of rTMS intervention $(p<0.05$, uncorrected). The significant differences were demonstrated in Figure 2 and detailed information of significant clusters and peak voxels was recorded in Table 2 . No significant changes were observed in the CT after a 10-day acupuncture and conventional medication treatment. Moreover, the quantitative diffusion FA value in the bilateral PLIC, M1, and SMA was significantly different between the RT and CT groups after rTMS and conventional treatment ( $p<0.05$, uncorrected) (Figure 3 and Table 3 ).

3.3. Relationship between Motor Improvement and White Microstructure. To evaluate the relationship between the quantitative FA values and the functional clinical recovery, we detected a significant and positive correlation between the altered FA value in the ipsilesional PLIC and changes in FMA scores in RT group $(r=0.78, p=0.039)$ (Figure 4). For the CT group, although positive correlation was found, it was not
TABLE 3: Brain regions with significant clusters and peak voxel coordinates showing FA difference between rTMS group and control group.

\begin{tabular}{|c|c|c|c|}
\hline Brain region & $\begin{array}{c}\text { MNI coordinates } \\
(x, y, z)\end{array}$ & $t$ value & $\begin{array}{c}\text { Voxel } \\
\text { number }\end{array}$ \\
\hline \multicolumn{4}{|l|}{$R T>C T$} \\
\hline Supp_motor_area_L & $0,-1,61$ & 7.91 & 294 \\
\hline Precentral_L & $-44,-13,60$ & 3.63 & 75 \\
\hline Precentral_R & $36,-23,58$ & 3.56 & 51 \\
\hline Postcentral_L & $-48,-26,54$ & 2.78 & 59 \\
\hline Paracentral_lobule_L & $-2,-31,60$ & 4.13 & 162 \\
\hline Precuneus_L & $-2,-47,60$ & 3.9 & 372 \\
\hline Frontal_inf_tri_R & $49,32,7$ & 4.13 & 58 \\
\hline Thalamus_L & $-23,-20,6$ & 2.68 & 51 \\
\hline Insula_R & $45,6,9$ & 2.96 & 46 \\
\hline Frontal_sup_medial_R & $2,59,3$ & 3.63 & 286 \\
\hline Temporal_mid_R & $65,-36,3$ & 3.56 & 76 \\
\hline Occipital_sup_R & $11,-96,4$ & 2.61 & 42 \\
\hline \multicolumn{4}{|l|}{$R T<C T$} \\
\hline Frontal_mid_R & $29,53,3$ & -4.23 & 122 \\
\hline Caudate_L & $-13,17,10$ & -2.39 & 58 \\
\hline Frontal_sup_L & $-14,67,2$ & -2.76 & 50 \\
\hline Frontal_mid_L & $-30,10,51$ & -3.1 & 29 \\
\hline
\end{tabular}

MNI: Montreal Neurological Institute; L: left; R: right; RT: repetitive transcranial magnetic stimulation treatment; CT: conventional treatment.

significant $(r=0.27, p=0.52)$. No significant correlation was observed in other areas.

\section{Discussion}

In this study, 10-day successive sessions of exciting high frequency $(10 \mathrm{~Hz})$ rTMS over the ipsilesional motor cortex were evaluated in acute ischemic stroke patients, as an add-on therapy to current therapy for its effect on motor function recovery. Voxel-based diffusion parameter analysis was applied to investigate the microstructural alteration after this treatment. The investigation showed that both RT plus CT (including acupuncture and medication) and CT alone improved the motor function of the affected limb. Furthermore, the improvement was greater in the RT + CT group than in the CT alone group as measured by FM score (Table 1). The significantly improved microstructural properties of motor-related white fibers and gray matter areas, which included the PLIC, M1, SMA, MFG, and CR, were also observed in the RT + CT group. The FA changes in the ipsilesional PLIC of rTMS-treated patients but not in that of control patients showed a linear relationship with the functional motor gains, suggesting a potential modulation of the motor function recovery and motor-related neural systems as well as the interhemispheric communication by repeated rTMS treatment in stroke patients. The results of this study also demonstrated that high frequency rTMS was 


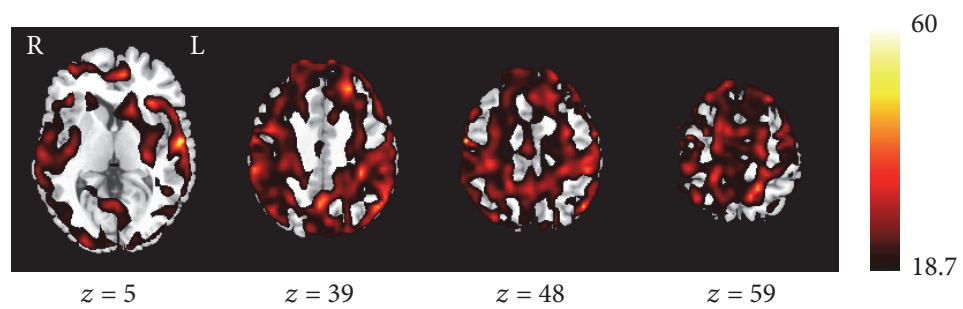

(a)

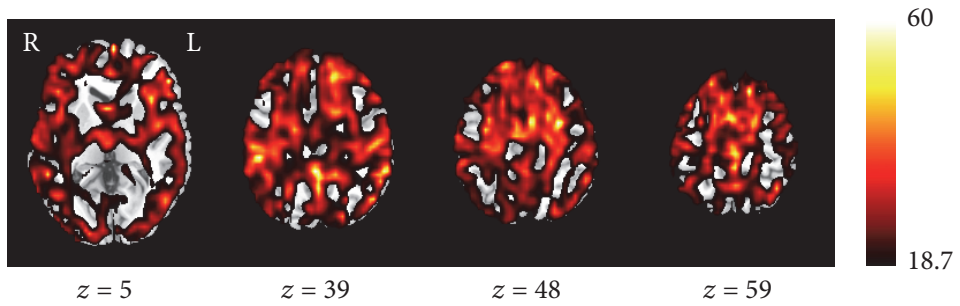

(b)

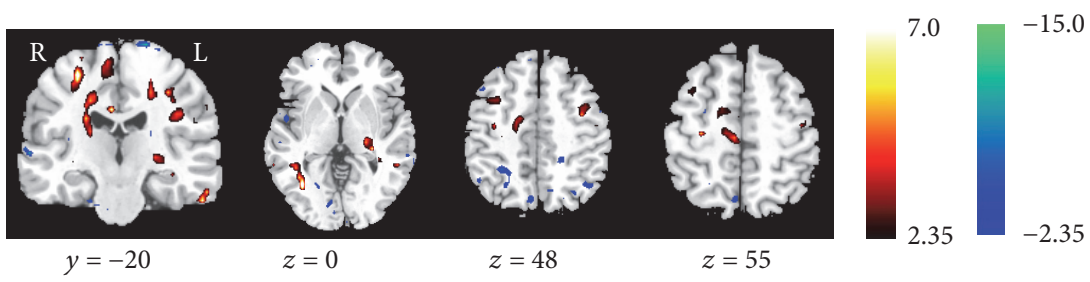

(c)
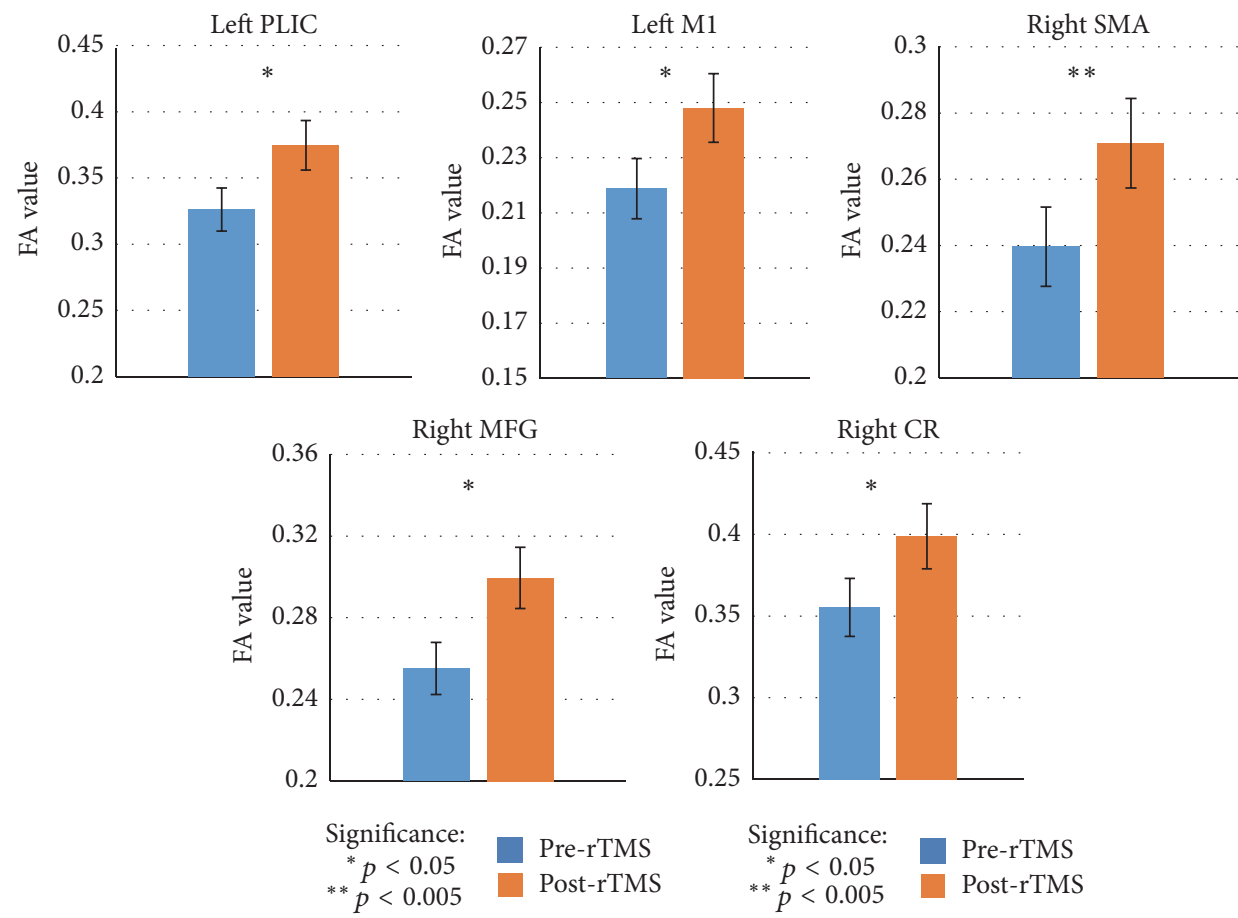

(d)

FIGURE 2: Comparison of FA maps between pre- and post-rTMS treatment for stroke patients in RT group. The FA results of one-sample $t$-test for stroke patients of pre- (a) and post-rTMS treatment (b). (c) Significantly changed brain areas are superimposed on the che2bet hemisphere of the Montreal Neurological Institute template brain in the three-view drawing $(p<0.05)$. The warm and cold tones separately indicate the increased and decreased FA value after rTMS treatment. (d) Bars represent the mean FA values. Vertical bars indicate estimated standard errors. Compared with the pre-rTMS treatment, the mean FA showed a significant increase after rTMS treatment in bilateral posterior limb of internal capsule (PLIC), left precentral gyrus (PG), right supplementary motor area (SMA), right middle frontal gyrus (MFG), and right corona radiate $(\mathrm{CR})$. 


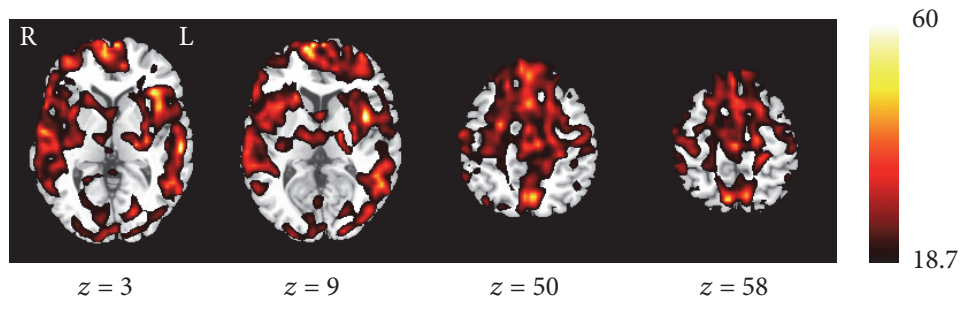

(a)

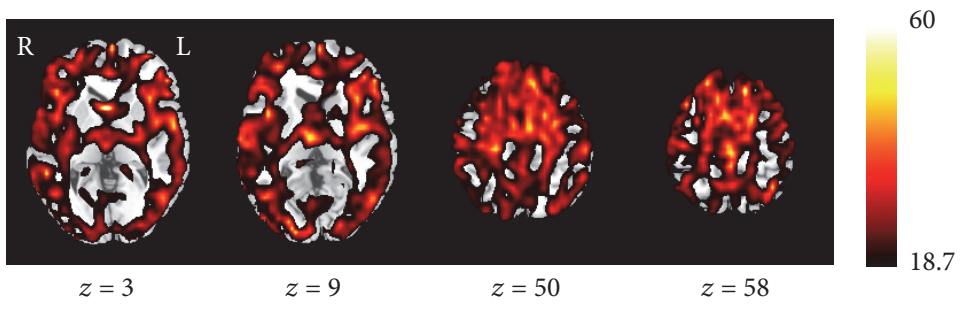

(b)

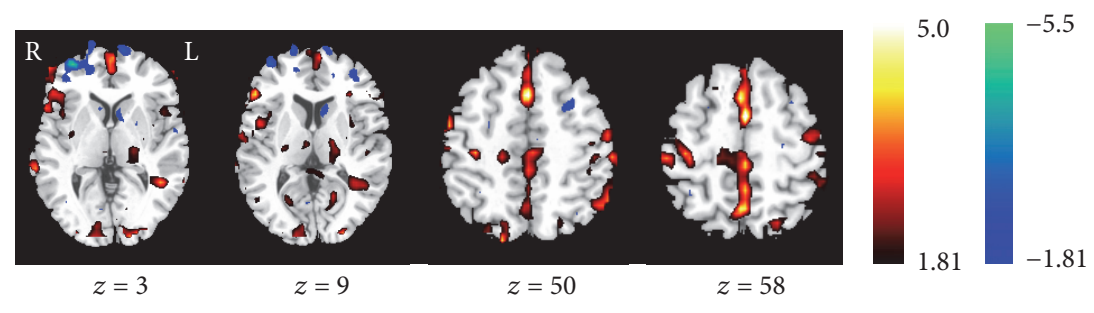

(c)
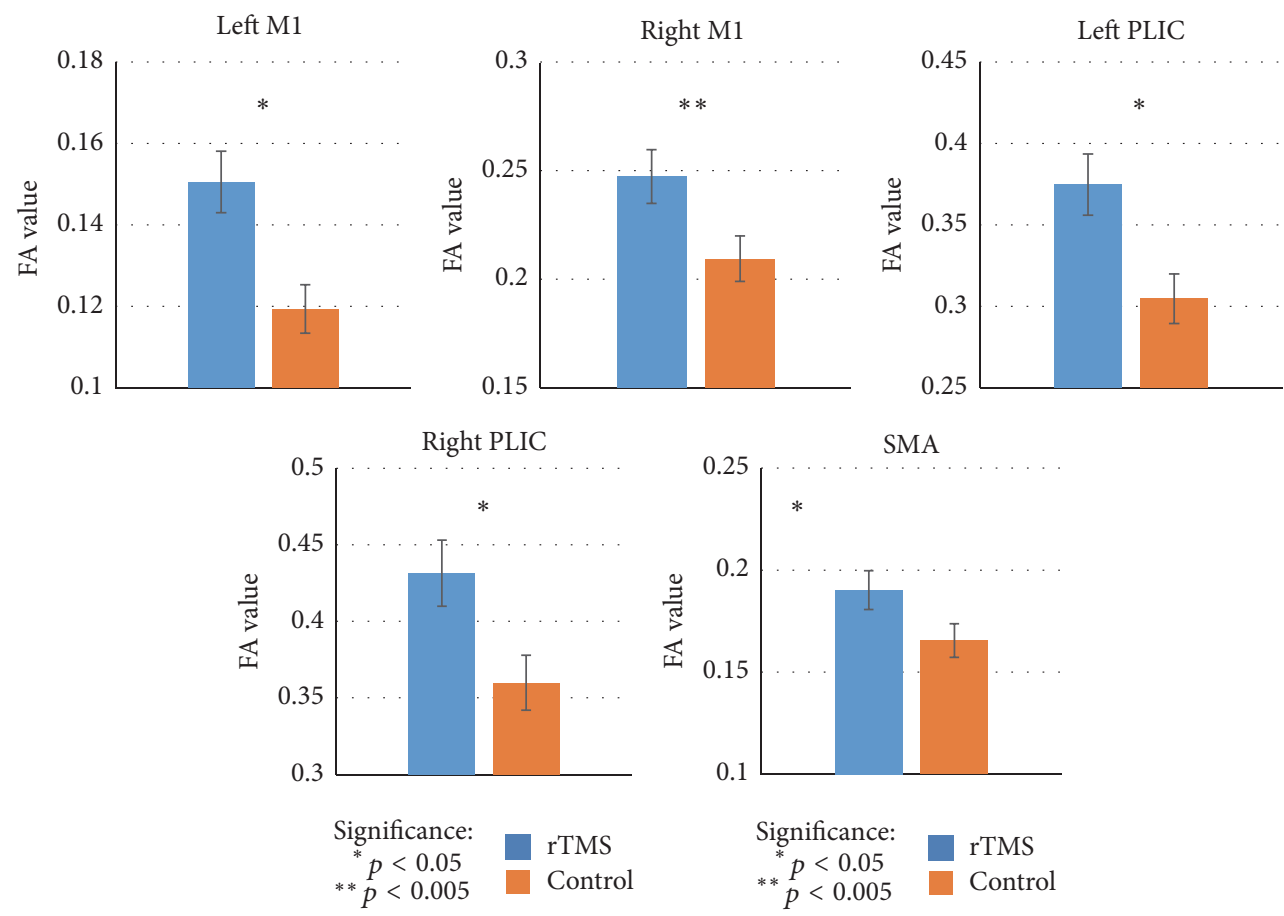

(d)

FIGURE 3: Comparison of FA maps between patients of RT and CT groups after treatment. The FA results of one-sample $t$-test for stroke patients of post-CT (a) and post-rTMS treatment (b). (c) Significantly changed brain areas are superimposed on the che2bet hemisphere of the Montreal Neurological Institute template brain in the three-view drawing $(p<0.05)$. The warm and cold tones separately indicate the increased and decreased FA value of RT. (d) Bars represent the mean FA values. Vertical bars indicate estimated standard errors. Compared with the CT, the mean FA value showed a significant increase after rTMS treatment in bilateral posterior limb of internal capsule (PLIC), primary motor area (M1), and supplementary motor area (SMA) in RT. 


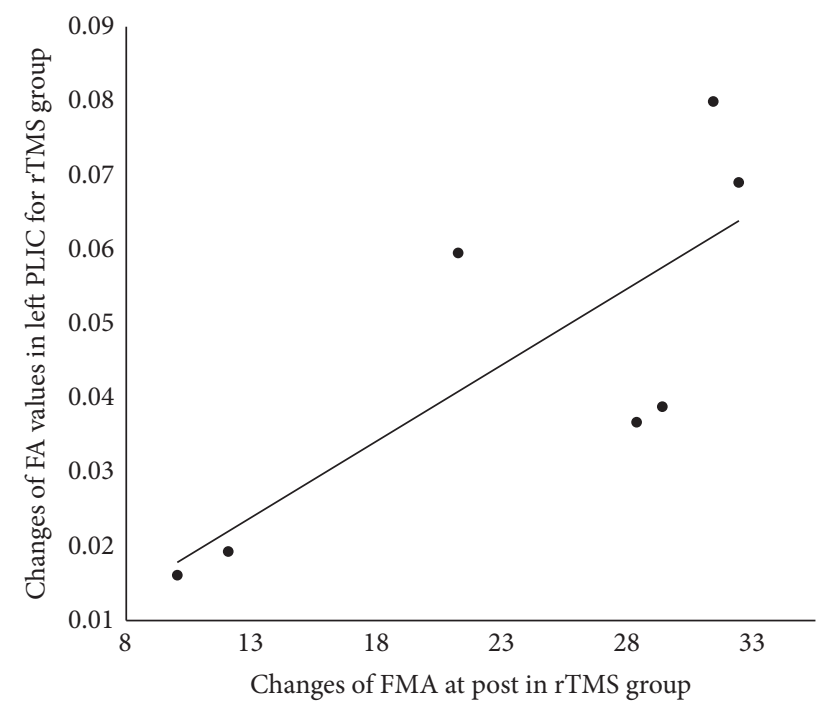

FIGURE 4: Relationship between changes of FA value in the ipsilesional posterior limb of internal capsule and changes of FMA score after rTMS treatment in the RT group (correlation coefficient $r=$ $0.78, p=0.039$ ).

a safe and tolerable add-on therapy for acute ischemic stroke patients.

Previous studies showed that rTMS alone was effective for motor recovery in stroke patients [14, 29-32]. In these studies, both single session $[14,29]$ and multiple sessions [30-32] of rTMS over affected hemisphere facilitated and enhanced motor recovery in stroke patients, in terms of movement accuracy [14], movement time [14], frequency of finger tapping [29], grip strength [30-32], and other motor aspects of motor function. However, so far, no study has reported FMA as a potential mechanism underlying high frequency rTMS-induced motor rehabilitation after stroke.

In this study, rTMS add-on therapy significantly increased FA values in the ipsilesional and contralesional PLIC and bilateral CR of stroke patients and this increase in the ipsilesional PLIC was positively correlated with the alteration of FMA scores. Although similar imaging results were observed in the CT group, they did not reach a significant level. These findings would reflect a boosting effect of rTMS on motor function recovery after stroke. Recent studies have shown that PLIC is mainly comprised of CST which control voluntary movement and is commonly used as the regions to the analysis of the integrity of CST $[33,34]$. Compared to other brain regions, PLIC would have a closer relationship with motor function. In addition, studies on stroke patients observed that degree of damage to the PLIC (reflected as reductions in FA values) is correlated with poor motor function [21], and the regional FA changes of PLIC could predict changes of motor impairment [35]. Thus, an increased FMA score would represent improved motor ability, and the positive correlation may indicate that the bigger FA value increased the better motor recovery of the patients.

There is evidence that PLIC and CR were mainly comprised of CST which project from the motor cortex to the
PLIC [34, 36, 37] and to brain stem [33]. Previous diffusion microstructural studies have showed significantly decreased FA values in ipsilesional $[5,38,39]$ and contralesional $[24,40]$ PLIC and CST after stroke. The degree of FA reduction in PLIC was also correlated with the damage of motor ability [5, 24]. During rehabilitation, the FA value of both the affected $[21,41]$ and unaffected CST increased significantly [42], and it has been used to predict the long-term motor outcome after stroke [21, 43]. Furthermore, a 7-day HF-rTMS reduced the infarct volumes, improved glucose metabolism, and inhibited neuronal apoptosis in lesional area in mice with acute experimental stroke [44]. In our study, we placed high frequency rTMS stimulation over the ipsilesional M1 area which is the origin of CST. Therefore, the neuromodulation of rTMS is possibly associated with or modulated through this structural pathway that involved improved energy metabolism.

Except for the white matter structures, we also observed increased FA value in several motor-related gray matter areas including the bilateral M1, SMA, ipsilesional thalamus, paracentral lobule, and contralesional MFG after rTMS treatment. It is known that the brain motor network, consisting of the M1, SMA, and paracentral lobule $[45,46]$, is crucially involved in the voluntary motor control [47]. Although MFG is not part of the motor network, previous functional MRI studies illustrated that MFG may monitor and reinforce motor performance and cognitive and executive functions during stroke recovery [48-51]. Besides, thalamus is involved in the modulation of motor function [52].

The cortical interhemispheric competition theory suggests a dual facilitation and inhibition modulation mechanism of the excitability of motor cortex between bilateral hemispheres that may underlie the recovery of motor dysfunction [53]. Indeed, increased FA value in CST and the transcallosal M1-M1 tracts was reported during stroke rehabilitation $[35,38]$, supporting that both are associated with subsequent motor recovery. A recent study of low frequency rTMS (LF-rTMS) on contralesional hemisphere in stroke patients with motor dysfunction suggests that LF-rTMS could increase FA value of transcallosal motor fibers and modulate and assist adaptive neuroplastic changes in stroke patients [54]. Together with this study's findings of FA value increased in bilateral M1 and motor-related cortex, it can be inferred that these findings may reflect the neuromodulation and therapeutic effects of rTMS on microstructural plasticity in stroke patients.

There are several limitations in the present study. First, only a small number of patients were included in each group that reduced the analysis power in statistical analysis. Second, regarding the behavioral assessment, only FMA, NIHSS, and BI were used to evaluate rTMS-induced motor recovery. Because FMA is mainly for motor function assessment, whereas NIHSS and BI are too general to evaluate specific motor ability, more refined and targeted motor measurement could produce more detailed findings. Third, the potential effects of acupuncture treatment and medication received by both RT and CT groups cannot be excluded. In addition, the longerterm effects of rTMS treatment were not evaluated.

Although both RT and CT improved the neurological scores, motor function, and daily activity ability, a greater 
improvement in FMA scores was observed in RT group than in the CT group, suggesting a specific effect of HFrTMS in this regard. A recent DTI study showed that a 4week acupuncture treatment with conventional medication could improve the FMA score and white matter diffusion parameters compared to medication alone in stroke patients with unilateral motor deficits [55]. Thus, the lack of clearcut different results between the RT $+\mathrm{CT}$ group and CT group in this study may have been obscured by the potential therapeutic effect of acupuncture present in both treatment groups, or the greater improvement in FMA score in the RT + CT group may still reflect additional beneficial effects of rTMS over CT on motor recovery. Future studies with separate controls of rTMS, acupuncture, and medication are needed to verify this possibility.

To our knowledge, this study is the first to have evaluated the effectiveness of high frequency rTMS add-on therapy in acute stroke patients with motor dysfunction using voxelbased diffusion parameter analysis. rTMS treatment plus CT for 10 days improved diffusion microstructures in motorrelated white matter and gray matter brain regions that correlated with the behavioral recovery in stroke patients. Our results also suggest that change in FA values of the ipsilesional PLIC could be a potential biomarker for motor recovery in stroke patient that deserves further investigation.

\section{Competing Interests}

The authors declare no competing interests regarding the publication of this paper.

\section{Acknowledgments}

This work was supported by the National Natural Science Foundation of China (no. 81271559) and the State Administration of Foreign Experts Affairs, China (nos. SZD201516 and SZD201606). The authors thank Professor Cheng Luo for his professional suggestion to their research.

\section{References}

[1] F. E. Buma, E. Lindeman, N. F. Ramsey, and G. Kwakkel, "Functional neuroimaging studies of early upper limb recovery after stroke: a systematic review of the literature," Neurorehabilitation and Neural Repair, vol. 24, no. 7, pp. 589-608, 2010.

[2] L. Liu, D. Wang, K. S. Lawrence Wong, and Y. Wang, "Stroke and stroke care in China huge burden, significant workload, and a national priority," Stroke, vol. 42, no. 12, pp. 3651-3654, 2011.

[3] A. K. Rehme and C. Grefkes, "Cerebral network disorders after stroke: evidence from imaging-based connectivity analyses of active and resting brain states in humans," The Journal of Physiology, vol. 591, no. 1, pp. 17-31, 2013.

[4] C. Grefkes and N. S. Ward, "Cortical reorganization after stroke: how much and how functional?" The Neuroscientist, vol. 20, no. 1, pp. 56-70, 2014.

[5] J. D. Schaechter, Z. P. Fricker, K. L. Perdue et al., "Microstructural status of ipsilesional and contralesional corticospinal tract correlates with motor skill in chronic stroke patients," Human Brain Mapping, vol. 30, no. 11, pp. 3461-3474, 2009.
[6] G. Liu, C. Dang, X. Chen et al., "Structural remodeling of white matter in the contralesional hemisphere is correlated with early motor recovery in patients with subcortical infarction," Restorative Neurology and Neuroscience, vol. 33, no. 3, pp. 309319, 2015.

[7] A. T. Barker, R. Jalinous, and I. L. Freeston, "Non-invasive magnetic stimulation of human motor cortex," The Lancet, vol. 325, no. 8437, pp. 1106-1107, 1985.

[8] M. Hallett, “Transcranial magnetic stimulation and the human brain," Nature, vol. 406, no. 6792, pp. 147-150, 2000.

[9] N. Takeuchi, T. Tada, M. Toshima et al., "Inhibition of the unaffected motor cortex by $1 \mathrm{~Hz}$ repetitive transcranial magnetic stimulation enhances motor performance and training effect of the paretic hand in patients with chronic stroke," Journal of Rehabilitation Medicine, vol. 40, no. 4, pp. 298-303, 2008.

[10] N. Takeuchi, T. Chuma, Y. Matsuo, I. Watanabe, and K. Ikoma, "Repetitive transcranial magnetic stimulation of contralesional primary motor cortex improves hand function after stroke," Stroke, vol. 36, no. 12, pp. 2681-2686, 2005.

[11] F. Fregni, P. S. Boggio, C. G. Mansur et al., "Transcranial direct current stimulation of the unaffected hemisphere in stroke patients," NeuroReport, vol. 16, no. 14, pp. 1551-1555, 2005.

[12] F. Hummel, P. Celnik, P. Giraux et al., "Effects of non-invasive cortical stimulation on skilled motor function in chronic stroke," Brain, vol. 128, no. 3, pp. 490-499, 2005.

[13] V. Di Lazzaro, P. Profice, F. Pilato et al., "Motor cortex plasticity predicts recovery in acute stroke," Cerebral Cortex, vol. 20, no. 7, pp. 1523-1528, 2010.

[14] Y.-H. Kim, S. H. You, M.-H. Ko et al., "Repetitive transcranial magnetic stimulation-induced corticomotor excitability and associated motor skill acquisition in chronic stroke," Stroke, vol. 37, no. 6, pp. 1471-1476, 2006.

[15] L. J. Lanyon, "Diffusion tensor imaging: structural connectivity insights, limitations and future directions," in NeuroimagingMethods, pp. 137-162, 2012.

[16] P. J. Basser and C. Pierpaoli, "Microstructural and physiological features of tissues elucidated by quantitative-diffusion-tensor MRI," Journal of Magnetic Resonance, Series B, vol. 111, no. 3, pp. 209-219, 1996.

[17] M. Møller, J. Frandsen, G. Andersen, A. Gjedde, P. VestergaardPoulsen, and L. Østergaard, "Dynamic changes in corticospinal tracts after stroke detected by fibretracking," Journal of Neurology, Neurosurgery, and Psychiatry, vol. 78, no. 6, pp. 587-592, 2007.

[18] J. Puig, S. Pedraza, G. Blasco et al., "Acute damage to the posterior limb of the internal capsule on diffusion tensor tractography as an early imaging predictor of motor outcome after stroke," American Journal of Neuroradiology, vol. 32, no. 5, pp. 857-863, 2011.

[19] A. Sterr, P. J. A. Dean, A. J. Szameitat, A. B. Conforto, and S. Shen, "Corticospinal tract integrity and lesion volume play different roles in chronic hemiparesis and its improvement through motor practice," Neurorehabilitation and Neural Repair, vol. 28, no. 4, pp. 335-343, 2014.

[20] S.-H. Cho, D. G. Kim, D.-S. Kim, Y.-H. Kim, C.-H. Lee, and S. H. Jang, "Motor outcome according to the integrity of the corticospinal tract determined by diffusion tensor tractography in the early stage of corona radiata infarct," Neuroscience Letters, vol. 426, no. 2, pp. 123-127, 2007.

[21] E. H. Kim, J. Lee, and S. H. Jang, "Motor outcome prediction using diffusion tensor tractography of the corticospinal tract in 
large middle cerebral artery territory infarct," NeuroRehabilitation, vol. 32, no. 3, pp. 583-590, 2013.

[22] S. H. Jang, K. Kim, S. H. Kim, S. M. Son, W. H. Jang, and H. G. Kwon, "The relation between motor function of stroke patients and diffusion tensor imaging findings for the corticospinal tract," Neuroscience Letters, vol. 572, pp. 1-6, 2014.

[23] Z. Liu, Y. Li, X. Zhang, S. Savant-Bhonsale, and M. Chopp, "Contralesional axonal remodeling of the corticospinal system in adult rats after stroke and bone marrow stromal cell treatment," Stroke, vol. 39, no. 9, pp. 2571-2577, 2008.

[24] C. Granziera, H. Ay, S. P. Koniak, G. Krueger, and A. G. Sorensen, "Diffusion tensor imaging shows structural remodeling of stroke mirror region: results from a pilot study," European Neurology, vol. 67, no. 6, pp. 370-376, 2012.

[25] E. M. Andrews, S.-Y. Tsai, S. C. Johnson et al., "Human adult bone marrow-derived somatic cell therapy results in functional recovery and axonal plasticity following stroke in the rat," Experimental Neurology, vol. 211, no. 2, pp. 588-592, 2008.

[26] C. G. Mansur, F. Fregni, P. S. Boggio et al., "A sham stimulationcontrolled trial of rTMS of the unaffected hemisphere in stroke patients," Neurology, vol. 64, no. 10, pp. 1802-1804, 2005.

[27] F. Fregni, P. S. Boggio, A. C. Valle et al., "A sham-controlled trial of a 5-day course of repetitive transcranial magnetic stimulation of the unaffected hemisphere in stroke patients," Stroke, vol. 37, no. 8, pp. 2115-2122, 2006.

[28] S. Groppa, A. Oliviero, A. Eisen et al., "A practical guide to diagnostic transcranial magnetic stimulation: report of an IFCN committee," Clinical Neurophysiology, vol. 123, no. 5, pp. 858882, 2012.

[29] M. Ameli, C. Grefkes, F. Kemper et al., "Differential effects of high-frequency repetitive transcranial magnetic stimulation over ipsilesional primary motor cortex in cortical and subcortical middle cerebral artery stroke," Annals of Neurology, vol. 66, no. 3, pp. 298-309, 2009.

[30] E. M. Khedr, A. E. Etraby, M. Hemeda, A. M. Nasef, and A. A. E. Razek, "Long-term effect of repetitive transcranial magnetic stimulation on motor function recovery after acute ischemic stroke," Acta Neurologica Scandinavica, vol. 121, no. 1, pp. 30-37, 2010.

[31] W. H. Chang, Y.-H. Kim, O. Y. Bang, S. T. Kim, Y. H. Park, and P. K. W. Lee, "Long-term effects of rTMS on motor recovery in patients after subacute stroke," Journal of Rehabilitation Medicine, vol. 42, no. 8, pp. 758-764, 2010.

[32] M. P. Malcolm, W. J. Triggs, K. E. Light et al., "Repetitive transcranial magnetic stimulation as an adjunct to constraintinduced therapy: an exploratory randomized controlled trial," American Journal of Physical Medicine and Rehabilitation, vol. 86, no. 9, pp. 707-715, 2007.

[33] J. K. Park, B. S. Kim, G. Choi et al., "Evaluation of the somatotopic organization of corticospinal tracts in the internal capsule and cerebral peduncle: results of diffusion-tensor MR tractography," Korean Journal of Radiology, vol. 9, no. 3, pp. 191195, 2008.

[34] K. A. Potter-Baker, N. M. Varnerin, D. A. Cunningham et al., "Influence of corticospinal tracts from higher order motor cortices on recruitment curve properties in stroke," Frontiers in Neuroscience, vol. 10, article 79, 2016.

[35] R. Lindenberg, L. L. Zhu, T. Rüber, and G. Schlaug, "Predicting functional motor potential in chronic stroke patients using diffusion tensor imaging," Human Brain Mapping, vol. 33, no. 5, pp. 1040-1051, 2012.
[36] D. A. Cunningham, A. Machado, D. Janini et al., "Assessment of inter-hemispheric imbalance using imaging and noninvasive brain stimulation in patients with chronic stroke," Archives of Physical Medicine and Rehabilitation, vol. 96, no. 4, pp. S94S103, 2015.

[37] A. I. Holodny, D. M. Gor, R. Watts, P. H. Gutin, and A. M. Ulug, "Diffusion-tensor MR tractography of somatotopic organization of corticospinal tracts in the internal capsule: initial anatomic results in contradistinction to prior reports," Radiology, vol. 234, no. 3, pp. 649-653, 2005.

[38] Y.-T. Fan, K.-C. Lin, H.-L. Liu, Y.-L. Chen, and C.-Y. Wu, "Changes in structural integrity are correlated with motor and functional recovery after post-stroke rehabilitation," Restorative Neurology and Neuroscience, vol. 33, no. 6, pp. 835-844, 2015.

[39] Y. Li, P. Wu, F. Liang, and W. Huang, "The microstructural status of the corpus callosum is associated with the degree of motor function and neurological deficit in stroke patients," PLOS ONE, vol. 10, no. 4, Article ID e0122615, 2015.

[40] S. Y. Kwak, S. S. Yeo, B. Y. Choi, C. H. Chang, and S. H. Jang, "Corticospinal tract change in the unaffected hemisphere at the early stage of intracerebral hemorrhage: a diffusion tensor tractography study," European Neurology, vol. 63, no. 3, pp. 149$153,2010$.

[41] Y. Takenobu, T. Hayashi, H. Moriwaki, K. Nagatsuka, H. Naritomi, and H. Fukuyama, "Motor recovery and microstructural change in rubro-spinal tract in subcortical stroke," NeuroImage: Clinical, vol. 4, pp. 201-208, 2014.

[42] K. Marumoto, T. Koyama, M. Hosomi et al., "Diffusion tensor imaging predicts the outcome of constraint-induced movement therapy in chronic infarction patients with hemiplegia: a pilot study," Restorative Neurology and Neuroscience, vol. 31, no. 4, pp. 387-396, 2013.

[43] J. Puig, G. Blasco, J. Daunis-I-Estadella et al., "Decreased corticospinal tract fractional anisotropy predicts long-term motor outcome after stroke," Stroke, vol. 44, no. 7, pp. 2016-2018, 2013.

[44] F. Gao, S. Wang, Y. Guo et al., "Protective effects of repetitive transcranial magnetic stimulation in a rat model of transient cerebral ischaemia: a microPET study," European Journal of Nuclear Medicine and Molecular Imaging, vol. 37, no. 5, pp. 954961, 2010.

[45] F. A. Middleton and P. L. Strick, "Basal ganglia and cerebellar loops: motor and cognitive circuits," Brain Research Reviews, vol. 31, no. 2-3, pp. 236-250, 2000.

[46] R. P. Dum and P. L. Strick, "Motor areas in the frontal lobe of the primate," Physiology \& Behavior, vol. 77, no. 4-5, pp. 677$682,2002$.

[47] H. Nakata, K. Sakamoto, A. Ferretti et al., "Negative BOLD effect on somato-motor inhibitory processing: an fMRI study," Neuroscience Letters, vol. 462, no. 2, pp. 101-104, 2009.

[48] J. Camchong, A. W. MacDonald III, C. Bell, B. A. Mueller, and K. O. Lim, "Altered functional and anatomical connectivity in schizophrenia," Schizophrenia Bulletin, vol. 37, no. 3, pp. 640650, 2011.

[49] K. Öneş, E. Y. Yalçinkaya, B. C. Toklu, and N. Caglar, "Effects of age, gender, and cognitive, functional and motor status on functional outcomes of stroke rehabilitation," NeuroRehabilitation, vol. 25, no. 4, pp. 241-249, 2009.

[50] A. W. S. Leung, S. K. W. Cheng, A. K. Y. Mak, K.-K. Leung, L. S. W. Li, and T. M. C. Lee, "Functional gain in hemorrhagic stroke patients is predicted by functional level and cognitive abilities 
measured at hospital admission," NeuroRehabilitation, vol. 27, no. 4, pp. 351-358, 2010.

[51] C.-H. Park, W. H. Chang, S. H. Ohn et al., "Longitudinal changes of resting-state functional connectivity during motor recovery after stroke," Stroke, vol. 42, no. 5, pp. 1357-1362, 2011.

[52] M. R. DeLong, M. D. Crutcher, and A. P. Georgopoulos, "Primate globus pallidus and subthalamic nucleus: functional organization," Journal of Neurophysiology, vol. 53, no. 2, pp. 530-543, 1985.

[53] A. C. Vidal, P. Banca, A. G. Pascoal, G. Cordeiro, J. SargentoFreitas, and M. Castelo-Branco, "Modulation of cortical interhemispheric interactions by motor facilitation or restraint," Neural Plasticity, vol. 2014, Article ID 210396, 8 pages, 2014.

[54] A. Demirtas-Tatlidede, M. Alonso-Alonso, R. P. Shetty, I. Ronen, A. Pascual-Leone, and F. Fregni, "Long-term effects of contralesional rTMS in severe stroke: safety, cortical excitability, and relationship with transcallosal motor fibers," NeuroRehabilitation, vol. 36, no. 1, pp. 51-59, 2015.

[55] Y. Li, Y. Wang, H. Zhang, P. Wu, and W. Huang, "The effect of acupuncture on the motor function and white matter microstructure in ischemic stroke patients," Evidence-Based Complementary and Alternative Medicine, vol. 2015, Article ID 164792, 10 pages, 2015. 

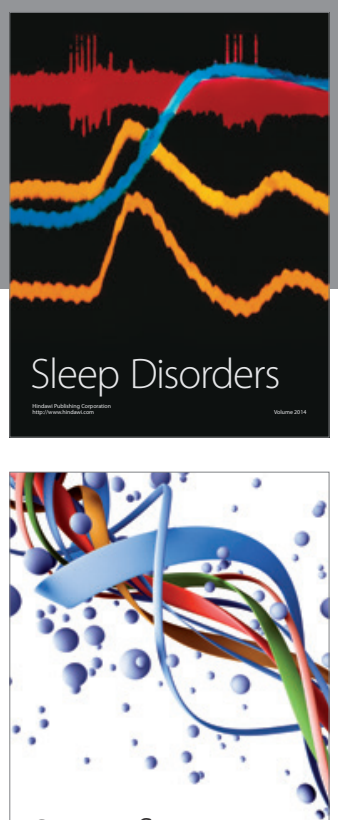

Scientifica
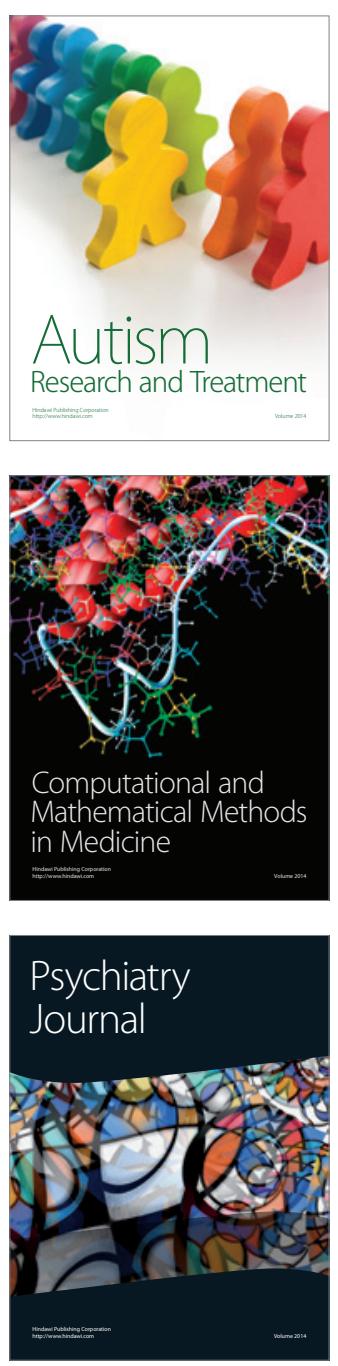
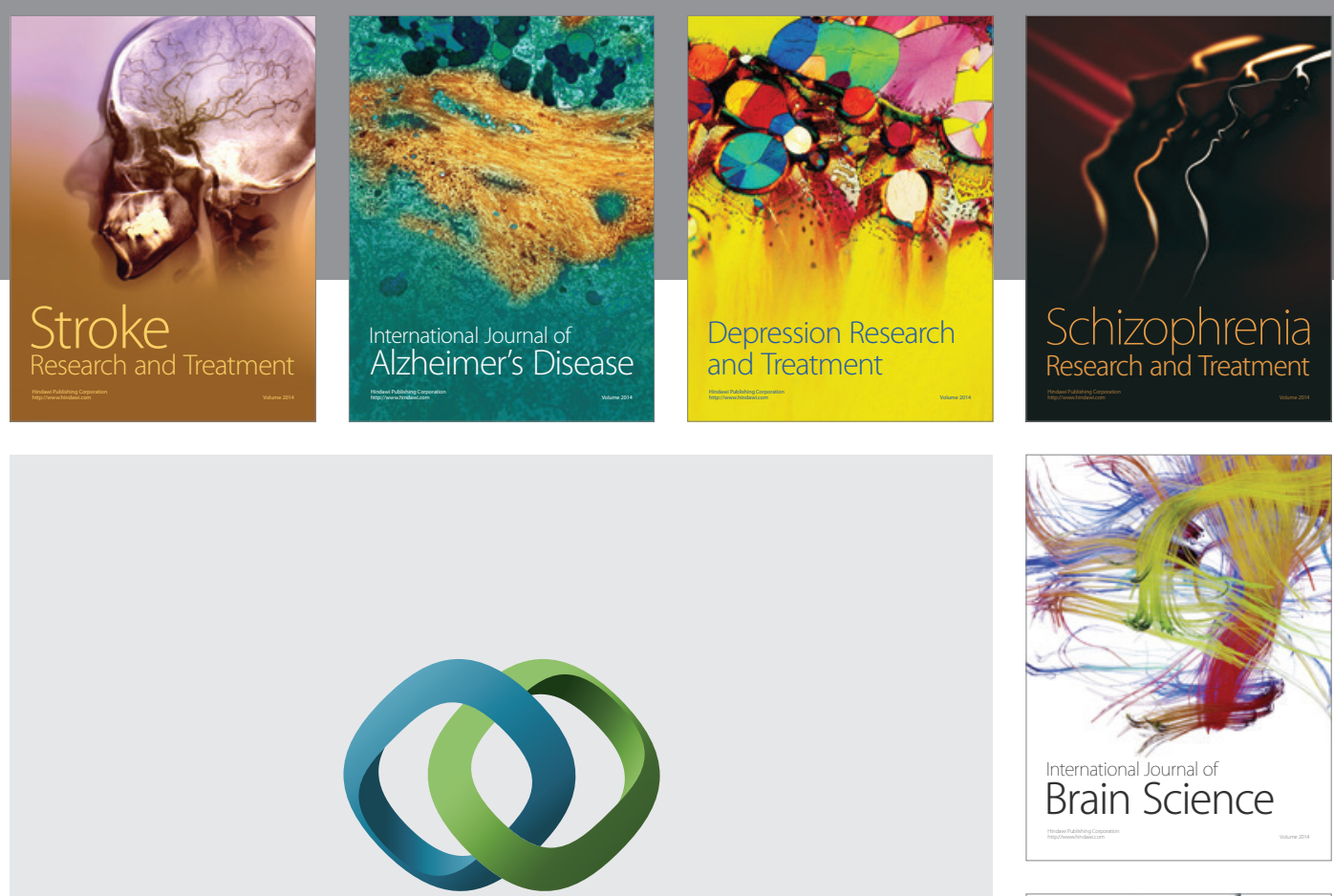

\section{Hindawi}

Submit your manuscripts at

http://www.hindawi.com
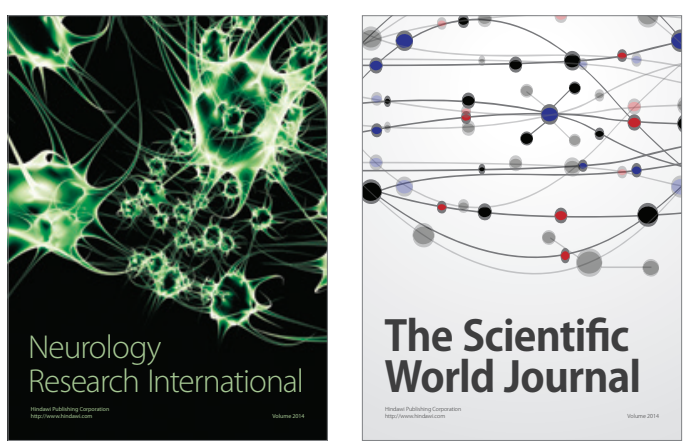

The Scientific World Journal

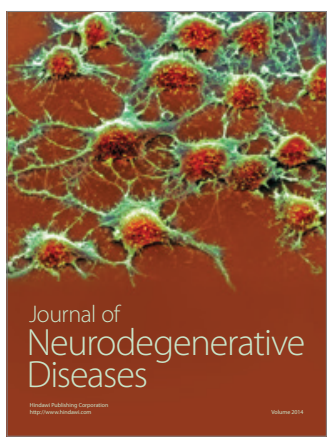

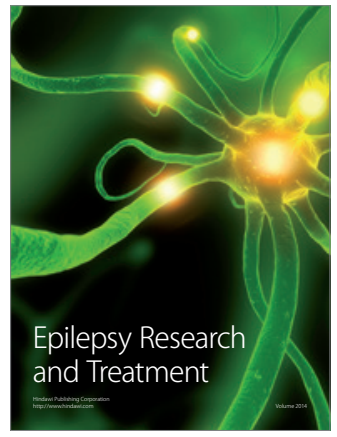

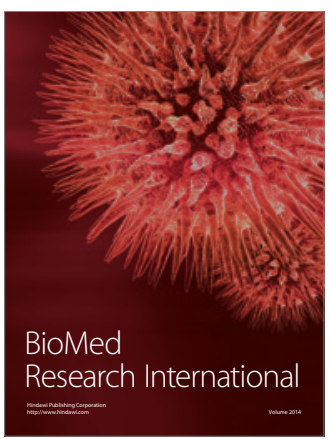

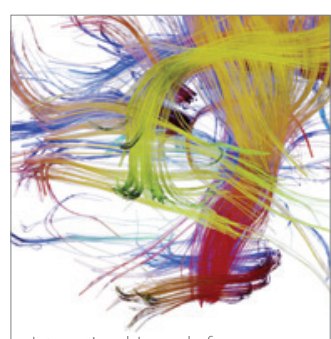

Brain Science

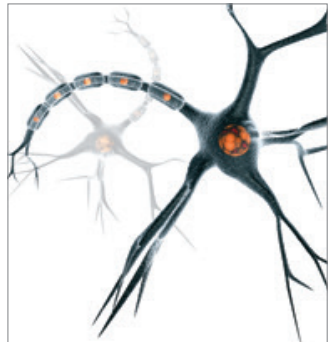

Neural Plasticity
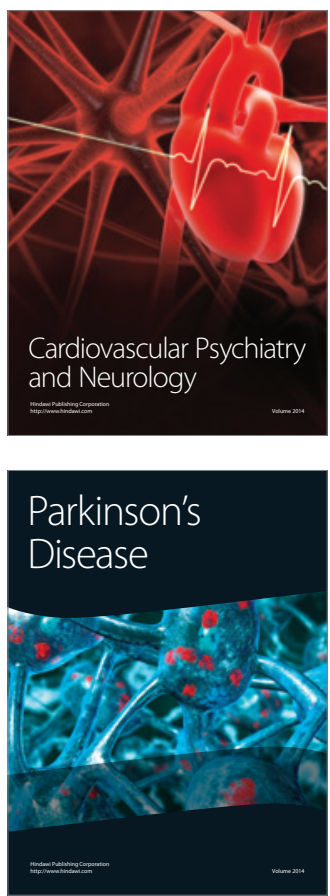